\title{
Synthesis and characterization of a new conducting polymer composite.
}

\begin{abstract}
Organic conductive composite films have been synthesized by electropolymerization of pyrrole in the presence of chitosan and p-toluene sulfonic acid sodium salt. The obtained conductive polymer composite films have been characterized by Fourier Transform Infrared spectroscopy, dynamic mechanical analysis, scanning electron microscopy, X-ray diffraction and conductivity measurements. The prepared polymer composite films had the amorphous structure and exhibited the enhanced conductivity and mechanical properties due to the presence of chitosan in the composite films.
\end{abstract}

Keyword: Conducting polymer; Chitosan; Toluene sulfonic acid sodium salt. 